\title{
Value of whole-body low-dose computed tomography in patients with ventriculoperitoneal shunts: a retrospective study
}

\author{
Andrej Pala, MD, ${ }^{1}$ Fadi Awad, MD, ${ }^{1}$ Michael Braun, MD, ${ }^{2}$ Michal Hlavac, MD, ${ }^{1}$ \\ Arthur Wunderlich, DSc, ${ }^{3,4}$ Bernd Schmitz, MD, ${ }^{2}$ Christian Rainer Wirtz, MD, ${ }^{1}$ and \\ Jan Coburger, PD DrMed ${ }^{1}$
}

Departments of ${ }^{1}$ Neurosurgery, ${ }^{2}$ Neuroradiology, and ${ }^{3}$ Radiology; and ${ }^{4}$ Section for Experimental Radiology, University of UIm, Günzburg, Germany

\begin{abstract}
OBJECTIVE The gold standard for evaluation of ventriculoperitoneal (VP) shunt position, dislocation, or disconnection is conventional radiography. Yet, assessment with this modality can be challenging because of low image quality and can result in repetitive radiation exposure with high fluctuation in the radiation dose. Recently, CT-based radiation doses have been significantly reduced by using low-dose protocols. Thus, whole-body low-dose CT (LDCT) has become applicable for routine use in VP shunt evaluation. The authors here compared image quality and approximate radiation dose between radiography and LDCT in patients with implanted VP shunt systems.
\end{abstract}

METHODS Ventriculoperitoneal shunt systems have been investigated with LDCT scanning at the authors' department since 2015. A consecutive series of 57 patients (70 investigations) treated between 2015 and 2016 was retrospectively assessed. A historical patient cohort that had been evaluated with radiography was compared with the LDCT patients in terms of radiation dose and image quality. Three independent observers evaluated projection of the valve pressure level and correct intraperitoneal position, as well as complete shunt projection, using a Likert-type scale of 1-5, where 1 indicated "not assessable" and 5 meant "assessable with high accuracy." Descriptive statistics and the Mann-Whitney U-test were used for analysis.

RESULTS Twenty-seven radiographs (38.6\%) and 43 LDCT scans (61.4\%) were analyzed. The median dose-length product (DLP) of the LDCT scans was $100 \mathrm{mGy} \cdot \mathrm{cm}$ (range $59.9-183 \mathrm{mGy} \cdot \mathrm{cm})$. The median total dose-area product (DAP) of the radiographic images was $3177 \mathrm{mGy} \cdot \mathrm{cm}^{2}$ (range $641-13,833 \mathrm{mGy} \cdot \mathrm{cm}^{2}$ ). The estimated effective dose (EED) was significantly lower with the LDCT scan $(p<0.001)$. The median EED was 4.93 and $1.90 \mathrm{mSv}$ for radiographs and LDCT, respectively. Significantly better identification of the abdominal position of the distal shunt catheter was achieved with LDCT $(p<0.001)$. Simultaneously, significantly improved visualization of the entire shunt system was realized with this technique $(p<0.001)$. On the contrary, identification of the valve settings was significantly worse with LDCT $(p<$ 0.001).

CONCLUSIONS Whole-body LDCT scanning allows good visualization of the distal catheter after VP shunt placement. Despite the fact that only a rough estimation of effective doses is possible in a direct comparison of LDCT and radiography, the data showed that shunt assessment via LDCT does not lead to greater radiation exposure. Thus, especially in difficult anatomical conditions, as in patients who have undergone multiple intraabdominal surgeries, have a high BMI, or are immobile, the use of LDCT shunt evaluation has high clinical value. Further data are needed to determine the value of LDCT for the evaluation of complications or radiation dose in pediatric patients.

https://thejns.org/doi/abs/10.3171/2017.7.JNS17476

KEY WORDS computed tomography; ventriculoperitoneal shunt; dose-area product; radiation exposure; dose-length product; effective dose; diagnostic technique

ABBREVIATIONS BMI = body mass index; DAP = dose-area product; DLP = dose-length product; EED = estimated effective dose; LDCT = low-dose CT; VP = ventriculoperitoneal.

SUBMITTED February 28, 2017. ACCEPTED July 7, 2017.

INCLUDE WHEN CITING Published online January 5, 2018; DOI: 10.3171/2017.7.JNS17476. 
I MPAIRED cerebrospinal fluid circulation resulting in hydrocephalus is a common complication that is routinely treated with the implantation of a ventriculoperitoneal (VP) shunt. ${ }^{2}$ Correct intraperitoneal placement of the catheter or identification of its disconnection is usually determined using combined $2 \mathrm{D}$ radiographic investigations of the skull, chest, and abdomen. Pediatric studies have reported shunt failure in up to $50 \%$ of cases within the 1st year. ${ }^{6}$ Repetitive radiation exposure increases the risk of malignancies and leads to higher medical costs. ${ }^{1}$ Therefore, a precise diagnostic tool that adequately visualizes the shunt catheter might reduce these risks. In an ex vivo model, excellent sensitivity and specificity with even lower radiation exposure following whole-body low-dose CT (LDCT) to detect complications related to VP shunts were reported by Othman et al. ${ }^{3}$ Given these convincing experimental findings, LDCT was introduced at our center starting in 2015. After the 1st year of experience, we compared the quality of shunt images as well as the approximate radiation exposure between radiography and LDCT.

\section{Methods}

\section{Patients and Assessment of Image Quality}

All data were retrospectively assessed. Since October 2015 at our clinic, LDCT scanning of the chest and abdomen has been routinely performed in all patients after implantation of a VP, ventriculoatrial, or lumbar shunt, as well as in the case of suspected shunt dysfunction. All LDCT scans in this cohort were compared with images from a historic cohort that had undergone radiographic evaluation in the period from December 2013 to December 2015. Three independent observers with 3 different experience levels (neurosurgical resident, junior consultant, and senior consultant) evaluated CT and radiographic images of the chest and abdomen. At the time of image evaluation, the observers were not aware of the clinical findings of patients. Projection of the valve pressure level and correct intraperitoneal catheter localization, as well as complete shunt projection, were evaluated on a Likert-type scale ranging from 1 to 5 , where 1 was "not assessable" and 5 was "assessable with high accuracy." All images were evaluated with Impax 6.0 software (AGFA-Gevaert N.V.).

\section{Radiation Exposure and Assessment of Radiation Dose}

Conventional radiographic images were all obtained on a Carestream DRX-Evolution radiography system (Carestream Health Deutschland $\mathrm{GmbH}$ ). This system uses the latest-generation flat-panel detector technique for image acquisition with an unsurpassed quantum detective efficiency of approximately $70 \%$. A mobile detector makes the workflow and patient positioning easier, especially in immobilized patients. A Philips Brilliance 40 CT scanner (Philips Healthcare Deutschland) was used to perform the CT scanning, which had a range from the skull base to the pelvis with the parameters of $140 \mathrm{kV}$ and $15 \mathrm{mAs}$. The dose-length product (DLP; $\mathrm{mGy} \cdot \mathrm{cm}$ ) was used for the CT scans, and the dose-area product (DAP; $\mathrm{dGy} \cdot \mathrm{cm}^{2}$ ) was documented for radiographic images. Both DLP and DAP data were derived as reported by the CT scanner or the flat-panel detector of the radiographic system. Depending on visualization of the shunt, 1 or 2 radiographs per series for skull, chest, and abdomen were obtained. Immobile patients were imaged in the lying position in bed. Effective dose estimation was based on a conversion factor that depends on body regions. ${ }^{4}$ Effective doses for chest and abdomen radiographs as well as for chest and abdomen LDCT scans were assessed and compared. The estimated effective doses (EEDs) for CT were defined according to the recommendations and conversion factors done by Prokop et al. (http://dr.delis.de/scripts/DosisRechner.html). ${ }^{4}$

\section{Data Analysis}

Statistical analysis was performed using SPSS 21.0 (Lead Technologies Inc.). Descriptive statistics and the Mann-Whitney U-test were used for analysis. A p value < 0.05 was statistically significant. The study was conducted according to the international Declaration of Helsinki.

\section{Results}

\section{Radiographic and CT Characteristics}

Consecutive patients who had undergone shunt implantation or in whom shunt malfunction was suspected were included in the study. No pediatric patients were included in this study. The data for 70 investigations (57 patients) were evaluated retrospectively. The median patient age was 59.5 years (range 19-81 years), and 25 patients $(43.9 \%)$ were males. Twenty-seven radiographs $(38.6 \%)$ and 43 LDCT scans $(61.4 \%)$ were analyzed. Five patients had undergone multiple CT scans, and 1 had undergone multiple radiographic investigations. Each of these multiple studies was obtained after surgical revision. In 1 case, 2 revisions were necessary. The median DLP of the LDCT scans was $100 \mathrm{mGy} \cdot \mathrm{cm}$ (range $59.9-183 \mathrm{mGy} \cdot \mathrm{cm}$ ). The median DAP for the abdomen radiographs was 21.4 $\mathrm{dGy} \cdot \mathrm{cm}^{2}$ (range $4.0-98.0 \mathrm{dGy} \cdot \mathrm{cm}^{2}$ ); for the thorax radiographs, $1.24 \mathrm{dGy} \cdot \mathrm{cm}^{2}$ (range $0-26 \mathrm{dGy} \cdot \mathrm{cm}^{2}$ ); and for the skull radiographs, $4.68 \mathrm{dGy} \cdot \mathrm{cm}^{2}$ (range $0-14 \mathrm{dGy} \cdot \mathrm{cm}^{2}$ ). The median total DAP was $3177 \mathrm{mGy}^{\circ} \mathrm{cm}^{2}$ (range 641$\left.13,833 \mathrm{mGy} \cdot \mathrm{cm}^{2}\right)$.

In 37 cases $(52.9 \%)$, the Medos-Hakim programmable valve (Codman) was implanted. In 12 surgeries the proGAV 1.0 and in 20 surgeries the proGav 2.0 were used (both Miethke). In 1 case a lumboperitoneal shunt was implanted (Miethke).

In our cohort, only 1 disconnection was found in the radiographic group. In 5 cases in the CT group, a dislocation was revealed on the CT images and confirmed intraoperatively (Fig. 1).

\section{Quality of Shunt Visualization}

Significantly better visualization and identification of the abdominal catheter was achieved with LDCT ( $\mathrm{p}<$ 0.001, Mann-Whitney U-test; Fig. 2). Simultaneously, significantly improved visualization of the entire shunt system was achieved with this technique $(p<0.001$, MannWhitney U-test). On the contrary, identification of the valve settings was significantly worse with LDCT $(\mathrm{p}<$ 0.001, Mann-Whitney U-test). 

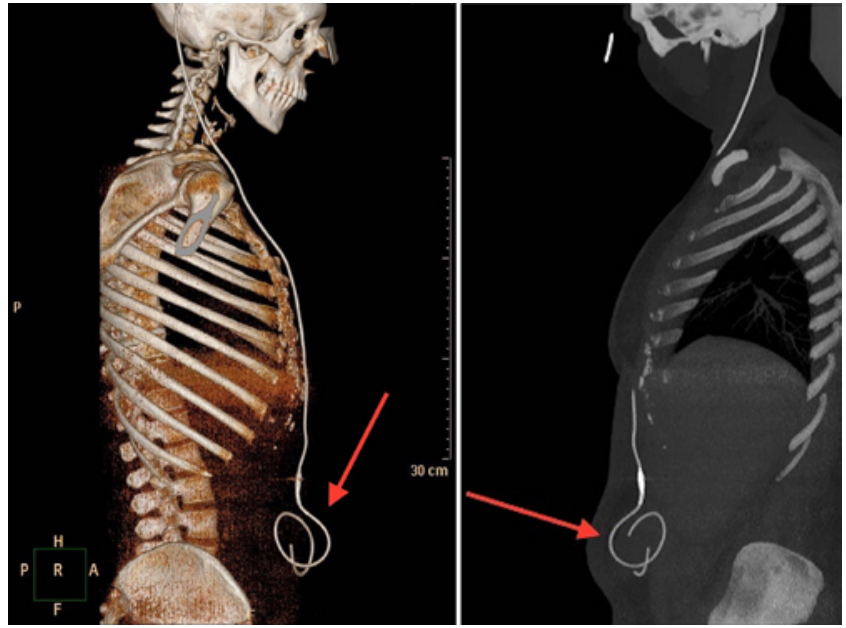

FIG. 1. Three-dimensional reconstruction of whole-body LDCT scan (left) and sagittal LDCT scan (right) showing dislocation of the abdominal catheter (arrows). Figure is available in color online only.

\section{Dose Estimation}

The median EEDs for radiographs and CT scans were $4.93 \mathrm{mSv}$ (range 1.06-24.03 mSv) and $1.90 \mathrm{mSv}$ (range 1.14-3.48 mSv), respectively. The EED of the LDCT scans was significantly lower than that of the radiographic images ( $\mathrm{p}<0.001$, Mann-Whitney U-test; Fig. 3).

\section{Illustrative Cases \\ Case 1}

A 68 -year-old woman with a body mass index (BMI) of $33.6 \mathrm{~kg} / \mathrm{m}^{2}$ underwent resection of an intraventricular subependymoma and developed communicating hydrocephalus. A VP shunt was implanted, and LDCT was performed after the surgery (Fig. 4). Despite the patient's high BMI, excellent image quality was achieved. The EED was with $1.73 \mathrm{mSv}$, which was even lower than the median EED for the entire cohort.

\section{Case 2}

A 77 -year-old man with a BMI of $33.8 \mathrm{~kg} / \mathrm{m}^{2}$ was admitted to our clinic with suspected normal-pressure hydrocephalus. Lumbar drain and clinical examination confirmed this diagnosis, and a VP shunt was implanted. To evaluate shunt disconnection or dislocation, conventional radiography was used (Figs. 5 and 6). The EED was 14.42 $\mathrm{mSv}$. The thorax and skull were examined in 1 plane, whereas 2 planes were needed for good evaluation of the distal catheter.

\section{Discussion}

In comparing radiographic images with whole-body LDCT scans, we investigated the quality of distal VP shunt depiction for clinical evaluation and approximate radiation exposure. Distal catheter failure in patients with VP shunts has been reported in up to $30 \%$ of cases. ${ }^{5}$ These patients are in need of repetitive radiographic examinations. Thus, cumulative radiation exposure increases. Othman et al. have confirmed significantly lower radiation exposure
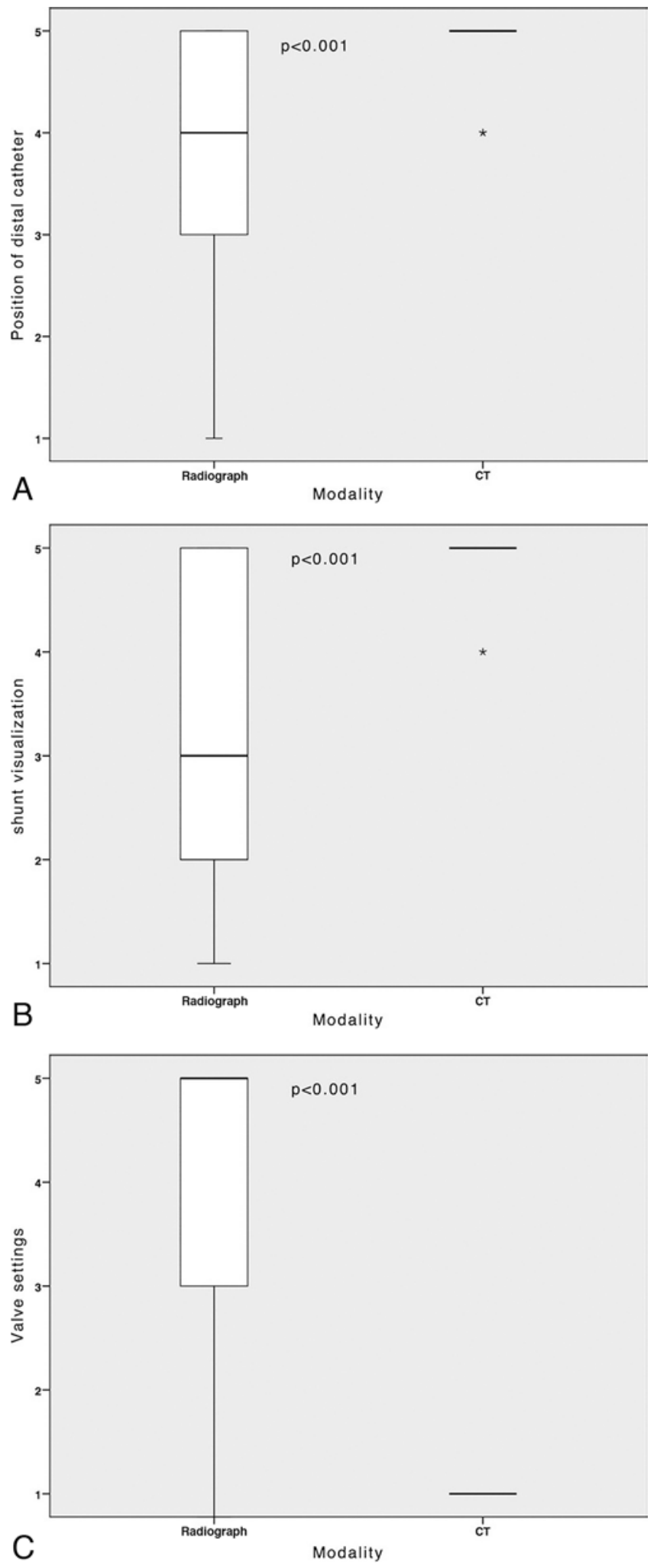

FIG. 2. Evaluation of the abdominal catheter position (A), shunt visualization (B), and valve settings (C) between radiography and LDCT, using a Likert scale of $1-5$, where 1 is "not assessable" and 5 is "assessable with high accuracy." Stars indicate the extreme value. 


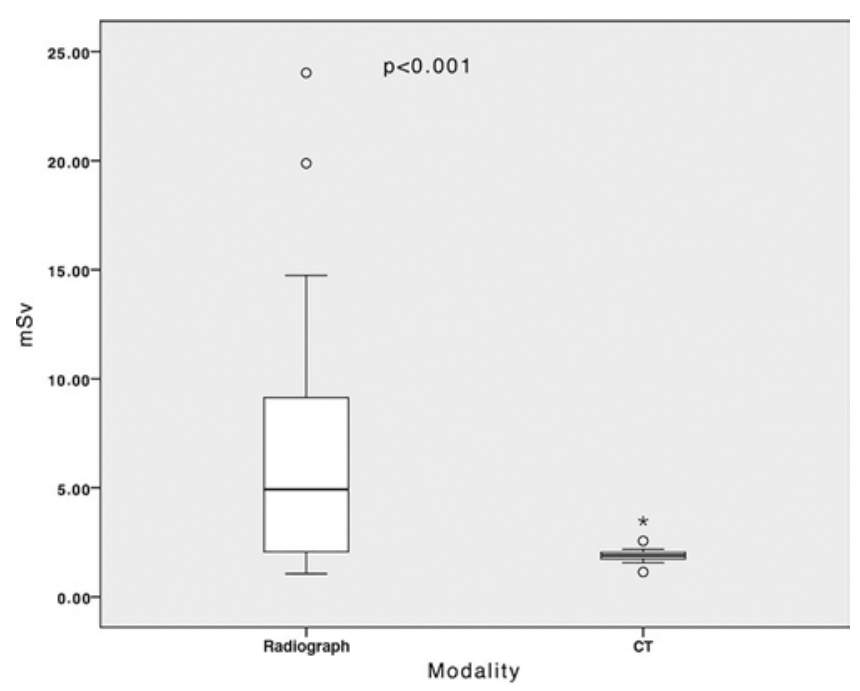

FIG. 3. Estimated effective dose (mSv) compared between radiography and LDCT. Star indicates the extreme value.

with whole-body LDCT in ex vivo models. ${ }^{3}$ Whole-body LDCT allowed good evaluation of the distal shunt catheter without the need for additional investigations in all cases in our study. The unambiguous visualization of the distal catheter is of great value in cases with difficult anatomical conditions, as in patients who have undergone multiple intraabdominal surgeries or have a high BMI.

According to our results, LDCT scanning of the chest and abdomen depicts the shunt system more precisely and with much less variation in the radiation dose, compared to conventional radiographic images. Consider, for comparison, that a typical brain CT scan requires a DLP of approximately $800-850 \mathrm{mGy} \cdot \mathrm{cm}$.

Obese patients are especially at risk for abdominal shunt dislocation and higher radiation doses due to the difficulty in evaluating the distal shunt catheter position. We observed extremely high variation in the radiation dose with abdominal radiographic images compared to that with whole-body LDCT scans. Obese patients exhibit higher DAPs, and as shown in our illustrative cases, the EED is much higher in patients with increased BMI. The establishment of LDCT might reduce radiation exposure and improve image quality in this patient group. Many patients who need a VP shunt system are unable to stand and could require imaging with the patients in bed, increasing the radiation dose as well as the risk of inferior images even more. This phenomenon is irrelevant in the case of wholebody LDCT scanning. Evaluation is simpler and can easily be combined with head CT, which is often needed for shunt evaluation as well. Early recognition of shunt malposition permits faster decision making about further treatment steps without the need for additional imaging.

A negative aspect of whole-body LDCT is in the depiction of the valve pressure level. Additional radiographs of the valve are needed in cases in which the pressure level cannot be assessed with noninvasive methods. On the contrary, however, this information is not always necessary and the additional radiation dose from the direct valve radiographic image is very low. Besides, most modern shunt
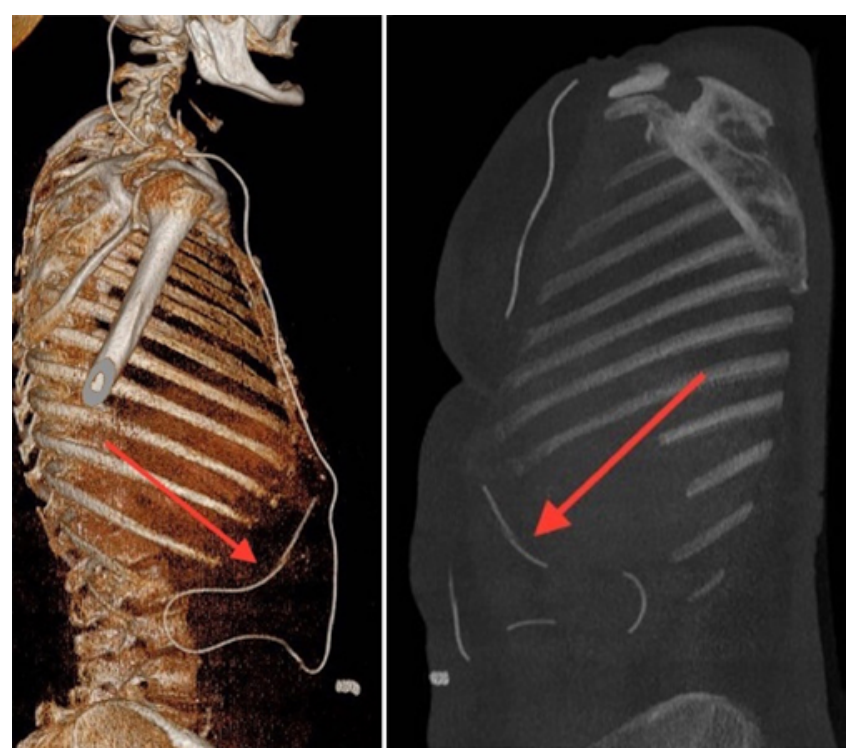

FIG. 4. Three-dimensional reconstruction of whole-body LDCT scan (left) and sagittal LDCT scan (right) showing the position of the VP shunt (arrows). Figure is available in color online only.

systems can be checked using other methods without additional radiation exposure, perhaps even in the scout view of a head CT scan.

Whole-body LDCT may be an imaging alternative that is not related to high radiographic exposure and provides excellent image quality for the evaluation of distal shunt failure.

\section{Challenges in the Assessment and Comparison of EED}

It is important to point out that it is methodologically impossible to directly compare the radiation exposure from CT scanning and radiography. Several factors hamper a direct radiation dose evaluation. With regard to the image acquisition technique, plain radiographic imaging uses cone beam radiation exposure in only one direction, whereas CT scanners use rotation-based radiation from all directions. This difference results in 2 different measurement units for radiation exposure. In plain radiographic images, exposure recordings are done by considering a radiated area with the DAP $\left(\mathrm{dGy} \cdot \mathrm{cm}^{2}\right)$. For CT scans, the radiation dose values are documented with the DLP, which describes the exposition of a volume over the whole scan length $(\mathrm{mGy} \cdot \mathrm{cm})$. The conversion factors are only empirically estimated coefficients that help to estimate the effective dose. ${ }^{4}$ Furthermore, the different organ radiation sensitivities and the involvement of different internal organs result in high variation in the effective dose. In our series, we did not differentiate between male and female patients. Thus, the EEDs in our series are only rough estimations. Especially concerning the radiographic images, EED may be even higher than estimated in our series. The modern flat-panel detector used in our department represents a standard radiographic device used by many clinics.

\section{Study Limitations}

Given the retrospective design of our study and low 


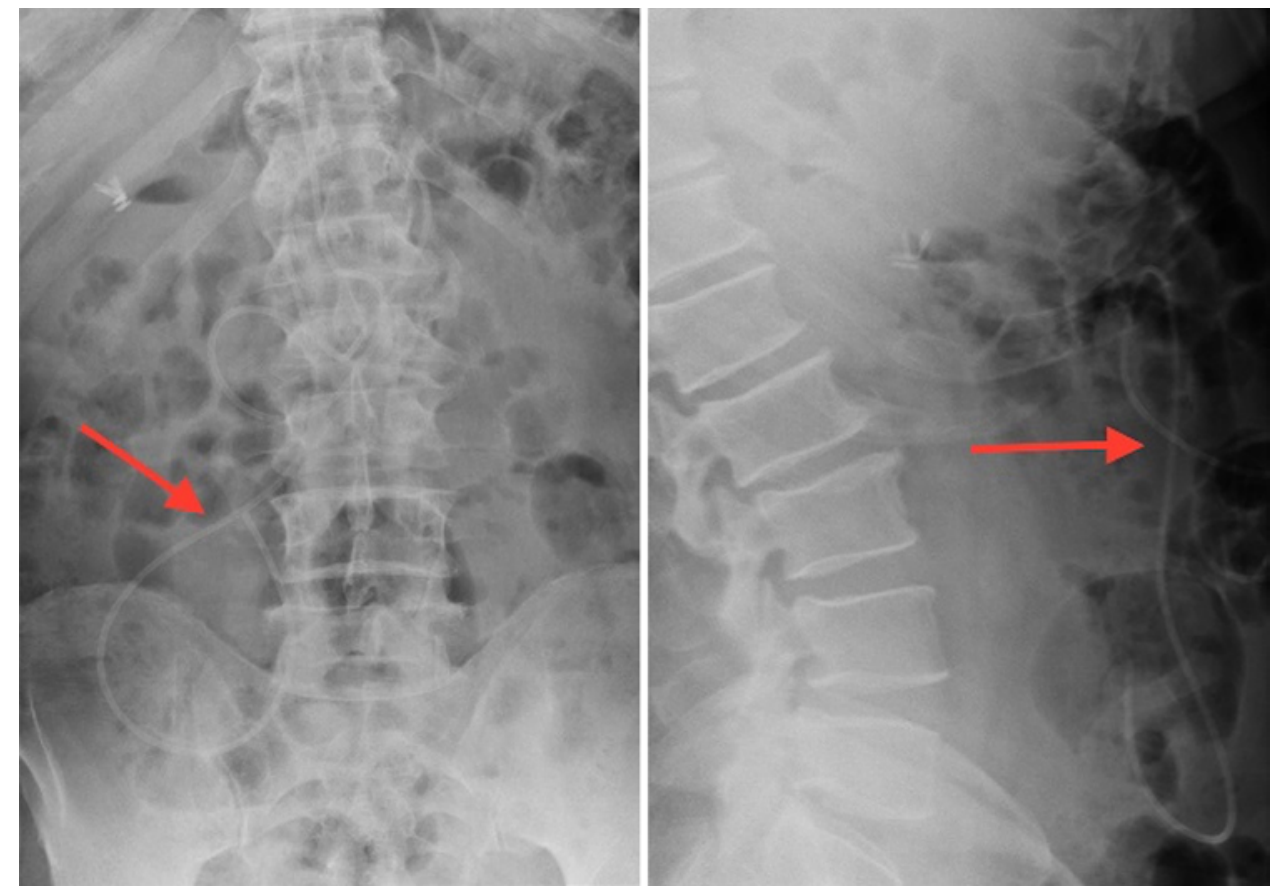

FIG. 5. Anteroposterior (left) and lateral (right) radiographic images of the abdomen showing the position of an abdominal catheter (arrows). Figure is available in color online only.

number of complications, we were unable to evaluate the role of whole-body LDCT in the assessment of complications such as disconnection. According to our data, however, excellent visualization of the distal shunt catheter is possible with LDCT, such that this technique could be superior to conventional radiography in the assessment of complications. Further studies are needed to answer this question. Our results are based on subjective assessments by 3 different observers with different experience levels. Our data may be biased in this regard. However, an assessment of image quality relevant to clinical decision making must be based mainly on subjective data.

\section{Future Perspectives and Clinical Implications}

Some studies dealing with pediatric hydrocephalic patients have compared LDCT and standard head CT and reported that a lower radiation dose provides adequate image quality for the recognition of intraventricular catheter location.? Alternatively, a low-dose head CT could be combined with whole-body LDCT and probably lead to even lower radiation doses and adequate image quality. However, further studies are necessary to evaluate this question.

No pediatric patients were included in our study. The benefit of whole-body LDCT seems to be mainly in the depiction of shunt systems in obese patients. As far as pediatric patients are concerned, we cannot draw any conclusions about that patient sample. Further studies including precise EED simulation models are necessary to clear up this question.

Future studies are planned to measure the exact effective dose emitted by different whole-body LDCT protocols and radiography using a standardized phantom with multiple dosimeters. But these measurements provide only standardized data. And the clinical situations of various body weights and difficult radiography conditions in immobile patients can only be assessed in a setup such as

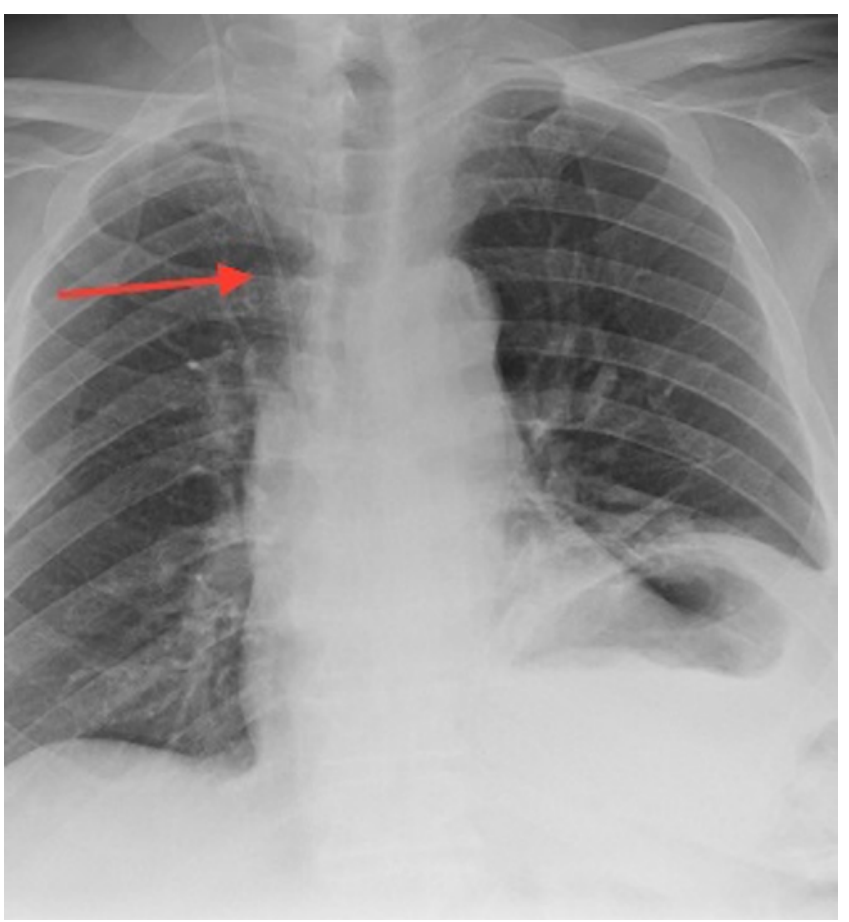

FIG. 6. Anteroposterior radiographic image of the chest depicting the position of the VP shunt system (arrow). Figure is available in color online only. 
that in the current study, which only allows for gross estimations of the effective dose.

\section{Conclusions}

Whole-body LDCT scanning of a VP shunt system can be easily applied in the routine workup for suspected shunt insufficiency, results in good depiction of the distal shunt catheter without additional examinations, and involves radiation exposure that is far lower than that with head CT. Computed tomography scanning of VP shunts does not seem to involve more effective radiation than radiographic studies, despite the methodological flaws in comparing radiation exposures between these different examinations in our study.

\section{References}

1. Fazel R, Krumholz HM, Wang Y, Ross JS, Chen J, Ting HH, et al: Exposure to low-dose ionizing radiation from medical imaging procedures. N Engl J Med 361:849-857, 2009

2. Lutz BR, Venkataraman P, Browd SR: New and improved ways to treat hydrocephalus: Pursuit of a smart shunt. Surg Neurol Int 4 (Suppl 1):S38-S50, 2013

3. Othman AE, Afat S, Hamou HA, Pjontek R, Tsiflikas I, Nikoubashman O, et al: High-pitch low-dose whole-body computed tomography for the assessment of ventriculoperitoneal shunts in a pediatric patient model: an experimental ex vivo study in rabbits. Invest Radiol 50:858-862, 2015

4. Prokop M: [Radiation dose in computed tomography. Risks and challenges.] Radiologe 48:229-242, 2008 (Ger)
5. Schucht P, Banz V, Trochsler M, Iff S, Krähenbühl AK, Reinert M, et al: Laparoscopically assisted ventriculoperitoneal shunt placement: a prospective randomized controlled trial. J Neurosurg 122: 1058-1067, 2015

6. Tuli S, Drake J, Lawless J, Wigg M, Lamberti-Pasculli M: Risk factors for repeated cerebrospinal shunt failures in pediatric patients with hydrocephalus. J Neurosurg 92:31-38, 2000

7. Udayasankar UK, Braithwaite K, Arvaniti M, Tudorascu D, Small WC, Little S, et al: Low-dose nonenhanced head CT protocol for follow-up evaluation of children with ventriculoperitoneal shunt: reduction of radiation and effect on image quality. AJNR Am J Neuroradiol 29:802-806, 2008

\section{Disclosures}

The authors report no conflicts of interest concerning the materials or methods used in this study or the findings specified in this paper.

\section{Author Contributions}

Conception and design: Pala, Coburger. Acquisition of data: Pala, Awad, Coburger. Analysis and interpretation of data: Pala, Braun, Wunderlich, Coburger. Drafting the article: Pala, Braun, Coburger. Critically revising the article: Braun, Hlavac, Schmitz, Wirtz. Reviewed submitted version of manuscript: Wunderlich. Statistical analysis: Pala, Coburger.

\section{Correspondence}

Andrej Pala: University of Ulm, Günzburg, Germany. andrej. pala@uni-ulm.de. 